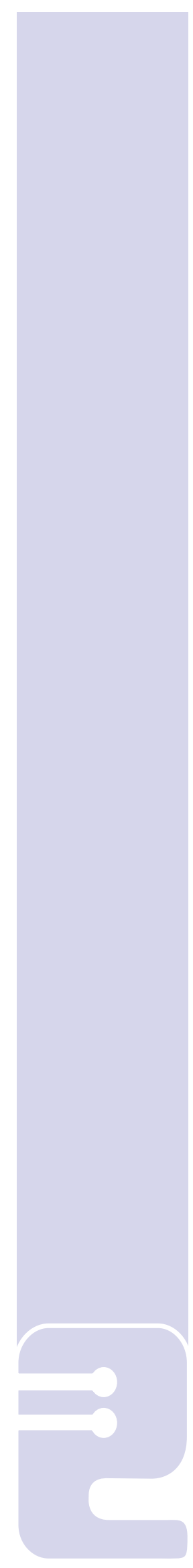

\title{
Diseño de estrategias y políticas de investigación en la educación bajo dinámica de sistemas
}

\author{
Milton M. Herrera R. ${ }^{1}$, Juliana Molano R. ${ }^{2}$, Harold Sandoval C. ${ }^{3}$
}

Recibido: Septiembre 03 de 2014 Aprobado: Diciembre 09 de 2014

\section{Resumen:}

Este artículo propone estrategias y políticas de investigación basadas en la capacidad de aprendizaje de las universidades (Know Why). Con la presente propuesta se busca fomentar la interacción entre los contextos empresariales, investigativo y académico. Por tanto el artículo se enfoca en la investigación como un proceso de creación e innovación basada en experiencias anteriores y en generación de conocimiento, las cuales son parte crucial de la estrategia que se propone.

El diseño de políticas y estrategias de investigación tiene inmersas unas cualidades sistémicas que lo caracterizan, es por ello que a través de la metodología propuesta desde la dinámica de sistemas, se analiza el comportamiento de la investigación de una universidad y se mide la estrategia de consolidación y aprovechamiento de las capacidades internas (Know How) y capacidades de aprendizaje (Know Why).

Palabras clave: Dinámica de sistemas, Gestión de la investigación, Educación, Diseño de políticas.

\section{Abstract:}

This article proposes research strategies and policies based on the learning capacity of the universities (Know Why). This proposal seeks to foster interaction among business, research and academic contexts. Therefore, the article focuses on research as a process of creation and innovation based on previous experiences and knowledge generation, which are crucial part of the strategy proposed.

Policy design and research strategies has immersed systemic topics that characterize them; through the proposed methodology from system dynamics, we analyze the research behavior of one university and we measure the achievement and consolidation strategy of internal capabilities (Know How) and learning skills (Know Why).

Keywords: System dynamics, Research management, Education, Policies design.

\footnotetext{
${ }^{1}$ Ingeniero de producción, magister en Ingeniería Industrial de la Universidad Distrital Francisco José de Caldas. Docente Investigador de la Corporación Universitaria Minuto de Dios - UNIMINUTO y Universidad Católica de Colombia. Semillero GETEC-FENIX

${ }^{2}$ Ingeniera Industrial y estudiante de maestría en gestión de organizaciones. Docente Corporación Universitaria Minuto de Dios - UNIMINUTO. Semillero FENIX

3 Ingeniero de mercados de la Universidad Piloto de Colombia. Joven investigador y líder estudiantil del Semillero SISTEMO. Áreas de interés: Dinámica de sistemas, trazabilidad e inteligencia de mercados.
} 


\section{INTRODUCCIÓN}

La Universidad juega un papel activo en el desarrollo del conocimiento a través de la investigación, mientras que el progreso de un país requiere de condiciones particulares centradas en la capacidad creativa que se gesta en los procesos investigativos y las políticas adoptadas por las instituciones privadas y públicas. La interacción de múltiples factores desenvuelve un comportamiento complejo en el que confluyen características particulares que dejan ver la relación Universidad-Empresa-Estado. Es así que la dinámica de sistemas permite la comprensión de dichas relaciones desde una óptica de sistema (un conjunto de elementos que se relacionan entre sí de manera tal que un cambio en uno de ellos modifica al conjunto). Este enfoque permite una visión clara y realista, donde se pueden analizar las relaciones entre los elementos que configuran la estructura que provoca el comportamiento que se desea estudiar, entender y en ocasiones modificar.

La acumulación de conocimientos y el desarrollo de capacidades de aprendizaje de las universidades se debe difundir de tal manera que se dé un impacto en el desarrollo social, político, económico, cultural y ambiental de un país, a través de los procesos investigativos. Para ello se deben proponer estrategias basadas en el reconocimiento del valor de los procesos investigativos y las capacidades internas de las universidades. Seguidamente, se proponen políticas que favorecen el ambiente investigativo soportado en la capacidad de aprendizaje adquirida en su quehacer. Estas capacidades de aprendizaje y conocimiento se convierten en elementos que permean la sociedad empresarial y estatal.
El artículo plantea estrategias encaminadas a propiciar las políticas adecuadas para el desarrollo de la investigación con un enfoque de competitividad y productividad, enmarcado en las capacidades de aprendizaje y conocimiento desarrolladas al interior de las universidades. Este diseño de políticas se fundamenta en el uso de la metodología de dinámica de sistemas, propuesta por los autores de este artículo. Se muestran los referentes a los diferentes modelos disponibles en cuanto a dinámica de sistemas se refiere y los datos de los grupos de investigación asociados a la universidad empleada en el caso de estudio, a través de los cuales se propone una hipótesis dinámica y un Diagrama Causal del comportamiento de la investigación, con el cual se pretenden plantear las estrategias y políticas de investigación para el caso de estudio en particular.

\section{COMPORTAMIENTO SISTÉMICO DE LA UNIVERSIDAD-EMPRESA-ESTADO}

En algunas ocasiones el Hombre orienta sus actividades a través de suposiciones, mitos o conjeturas que él crea o desarrolla sin argumentos validados desde la realidad (Herrera \& Cortes, 2011). En contraposición que la investigación científica es un proceso creativo que conlleva una transformación del entorno y un contexto soportado desde la realidad. En el desarrollo de los procesos investigativos confluyen tres actores (Universidad-Empresa-Estado) que interactúan y hacen de este proceso un sistema que presenta cierta complejidad de relación. Es por ello que por medio de la dinámica de sistemas algunos autores plantean estrategias y políticas educativas en este sentido. Algunos trabajos desarrollados desde el enfoque de dinámica de sistemas se presentan en la Tabla 1.

Tabla 1. Atecedentes de educación con un enfoque bajo dinámica de sistemas

\begin{tabular}{|c|c|c|c|}
\hline AUTOR(ES) & PAPER & AÑO & LUGAR \\
\hline $\begin{array}{l}\text { Davidsen Pal, Bjurklo Margaretha and } \\
\text { Wikstrom Hugo }\end{array}$ & $\begin{array}{l}\text { Introducing system dynamics in shools: the Nordic } \\
\text { experience }\end{array}$ & 1993 & Noruega \\
\hline Saeed Khalid & $\begin{array}{l}\text { Bringing experimental learning to social science: } \\
\text { simulation laboratory on issues of economic } \\
\text { development }\end{array}$ & 1993 & Tailandia \\
\hline Simons Kenneth & New technologies in simulation games & 1993 & $\begin{array}{l}\text { Estados } \\
\text { Unidos }\end{array}$ \\
\hline Richmond Barry & $\begin{array}{l}\text { Systems thinking: critical thinking skills for the } 1990 \text { s } \\
\text { and beyond }\end{array}$ & 1993 & $\begin{array}{l}\text { Estados } \\
\text { Unidos }\end{array}$ \\
\hline Brammer Richard and Viehweger Bernd & The player number growth in Social Browser Games & 2010 & Alemania \\
\hline
\end{tabular}




\begin{tabular}{|c|c|c|c|}
\hline AUTOR(ES) & PAPER & AÑO & LUGAR \\
\hline $\begin{array}{l}\text { Maldonado M. U., Maldonado M., } \\
\text { Chaim R. Pietrobon R. and Varvakis G. }\end{array}$ & $\begin{array}{l}\text { University Industry partherships with a focus on } \\
\text { BRIC countries: A systems perspective on the good, } \\
\text { the bad and the ugly }\end{array}$ & 2010 & $\begin{array}{l}\text { Brasil, } \\
\text { Italia y } \\
\text { Estados } \\
\text { Unidos }\end{array}$ \\
\hline $\begin{array}{l}\text { Abdelgawad A., Snaprud M. and } \\
\text { Krogstie J. }\end{array}$ & $\begin{array}{l}\text { Accessibility of Norwegian Municipalities Websites: } \\
\text { A qualitative System Dynamics Approach }\end{array}$ & 2010 & Noruega \\
\hline Cuménal Didier & $\begin{array}{l}\text { How the concept of organizational value highlights } \\
\text { corporate performance drivers a system dynamic } \\
\text { teaching model }\end{array}$ & 2010 & Francia \\
\hline $\begin{array}{l}\text { Díaz Adriana, Bucheli Victor and } \\
\text { Zarama Roberto }\end{array}$ & $\begin{array}{l}\text { La investigación en la Universidad de los Andes } \\
\text { como procesos de acumulación de capital humano: } \\
\text { una aproximación desde la dinámica de sistemas }\end{array}$ & 2010 & Colombia \\
\hline $\begin{array}{l}\text { Andrade Hugo, Navas Ximena and } \\
\text { Maestre Gina }\end{array}$ & $\begin{array}{l}\text { Prácticas y aprendizajes con la dinámica de } \\
\text { sistemas en la escuela Colombiana }\end{array}$ & 2010 & Colombia \\
\hline $\begin{array}{l}\text { Ruiz María, Trujillo Liliana and } \\
\text { Quintero Luis }\end{array}$ & $\begin{array}{l}\text { Enseñanza del concepto de función a partir de la } \\
\text { modelación matemática }\end{array}$ & 2010 & Colombia \\
\hline López Giovanni and Andrade Hugo & $\begin{array}{l}\text { Los especialistas y la integración de la DS en la } \\
\text { escuela: una propuesta de aprendizaje colaborativo }\end{array}$ & 2010 & Colombia \\
\hline $\begin{array}{l}\text { Rubiano Oscar, Cortes Mónica, Ramírez } \\
\text { Paulo and Trujillo Jasson }\end{array}$ & $\begin{array}{l}\text { Estudio de la planeación y desarrollo del proceso de } \\
\text { formación profesional integral desde el enfoque del } \\
\text { pensamiento sistémico }\end{array}$ & 2010 & Colombia \\
\hline $\begin{array}{l}\text { Cabana Arisitides, Fabra Henry, Sierra } \\
\text { Juan and Pontones Janer }\end{array}$ & $\begin{array}{l}\text { Efecto de las políticas de ampliación de cobertura } \\
\text { de la educación superior Colombiana. Una } \\
\text { perspectiva desde la dinámica de sistemas }\end{array}$ & 2010 & Colombia \\
\hline $\begin{array}{l}\text { Pineda Eliécer, Tellez Freddy and } \\
\text { Landazabal Diana }\end{array}$ & $\begin{array}{l}\text { Dinámica de sistemas, argumentación del conoci- } \\
\text { miento como apoyo en clubes de matemáticas }\end{array}$ & 2010 & Colombia \\
\hline $\begin{array}{l}\text { Soto Diego, Correa Christian and Parra } \\
\text { Favio }\end{array}$ & $\begin{array}{l}\text { Calificaciones, dinámica del estudio Grades, } \\
\text { student`s dynamics }\end{array}$ & 2010 & Colombia \\
\hline Carmona Guillermo and Montoya Juan & $\begin{array}{l}\text { Juego empresarial para la enseñanza de inventarios } \\
\text { con demanda variable }\end{array}$ & 2010 & Colombia \\
\hline $\begin{array}{l}\text { Castro Diego, Jaén Sebastián, Quiroz } \\
\text { Andrea, Duque Samuel and Ospina } \\
\text { Diego }\end{array}$ & $\begin{array}{l}\text { En busca de la utopía: plasmando un ideal que } \\
\text { puede ser real }\end{array}$ & 2010 & Colombia \\
\hline $\begin{array}{l}\text { Andersen J., LaVigne A., Radzicki M., } \\
\text { Richarson G. and Stuntz L. }\end{array}$ & $\begin{array}{l}\text { Teaching Characteristics of Complex Systems in } \\
\mathrm{K}-12 \text { Education }\end{array}$ & 2011 & $\begin{array}{l}\text { Estados } \\
\text { Unidos }\end{array}$ \\
\hline Beall Allyson and Ford Andrew & $\begin{array}{l}\text { Learning from system dynamics simulations: time } \\
\text { compression in system dynamics versus time } \\
\text { dilation in power system simulators }\end{array}$ & 2011 & $\begin{array}{l}\text { Estados } \\
\text { Unidos }\end{array}$ \\
\hline $\begin{array}{l}\text { Andrade H., Maestre Gina and López } \\
\text { Giovanni }\end{array}$ & $\begin{array}{l}\text { Desarrollo de competencias en la toma de } \\
\text { decisiones con dinámica de sistemas: una } \\
\text { experiencia en el aula }\end{array}$ & 2011 & Colombia \\
\hline
\end{tabular}




\begin{tabular}{|c|c|c|c|}
\hline AUTOR(ES) & PAPER & AÑO & LUGAR \\
\hline Arismendi José and Andrade Hugo & $\begin{array}{l}\text { Reflexiones sobre el diseño de experimentos con } \\
\text { dinámica de sistemas en educación }\end{array}$ & 2011 & Colombia \\
\hline $\begin{array}{l}\text { Díaz Adriana, Bucheli Victor and } \\
\text { Zarama Roberto }\end{array}$ & $\begin{array}{l}\text { Producción científica y acumulación de capital } \\
\text { humano: un modelo para estudiar su relación en } \\
\text { una universidad colombiana }\end{array}$ & 2011 & Colombia \\
\hline $\begin{array}{l}\text { López Giovanni, Andrade Hugo and } \\
\text { Maestre Gina }\end{array}$ & $\begin{array}{l}\text { Una experiencia de investigación- acción en la } \\
\text { integración de la DS en la escuela }\end{array}$ & 2011 & Colombia \\
\hline $\begin{array}{l}\text { Andrade Jair, Andrade Hugo and Gómez } \\
\text { Urbano }\end{array}$ & $\begin{array}{l}\text { Mejorando el proceso de aprendizaje de los } \\
\text { principios de la macroeconomía usando el modelo } \\
\text { económico clásico como herramienta pedagógica }\end{array}$ & 2011 & Colombia \\
\hline Ballesteros Ricaurte Javier & $\begin{array}{l}\text { Competencias de los estudiantes de ingeniería de } \\
\text { sistemas, análisis desde la dinámica de sistemas }\end{array}$ & 2011 & Colombia \\
\hline $\begin{array}{l}\text { Ramírez Ángela, Robayo Jenny and } \\
\text { Sotaquira Ricardo }\end{array}$ & $\begin{array}{l}\text { Nuevas formas de inmersión para juegos de } \\
\text { simulación en dinámica de sistemas }\end{array}$ & 2011 & Colombia \\
\hline \multicolumn{4}{|l|}{ Fuente: Elaboración propia } \\
\hline
\end{tabular}

En dichos trabajos se puede evidenciar que se permea la actividad educadora en el ámbito productivo de las compañías y que aquellos trabajos se encuentran enfocados al pensamiento sistémico e integral del futuro profesional. Richmond (1993) plantea una reflexión del pensamiento sistémico en la educación en Estados Unidos. A partir de este trabajo se generan propuestas como las de Davidsen et al. (1993), Saeed (1993) y Simons (1993) que utilizan la dinámica de sistemas como una metodología implementada en las actividades de educación que contribuyen en los procesos y análisis del aprendizaje.

Por otra parte, estudios como los de Díaz et al. (2010), Maldonado et al. (2010), López et al. (2011) y Richmond (1993), desarrollan desde el enfoque de la dinámica de sistemas la relación de la producción de conocimiento en la educación, proceso que se caracteriza por la interacción de diferentes actores y variables que complejizan su comprensión. La propuesta en este artículo se basa en el desarrollo descrito por Díaz et al. (2011), donde se muestra la interacción de variables que hacen posible el desarrollo del conocimiento en la Universidad, en un ambiente favorable de producción de conocimiento. En este sentido Andrade et al. (2010), Ruíz (2010), López \& Andrade (2010), Pineda (2010), Carmona (2010) y Andrade et al. (2011) muestran la intervención de la metodología desde el pensamiento sistémico en la educación.
Trabajos como el expuesto por Soto et al. (2010) y Ballesteros (2011) desarrollan desde el enfoque de dinámica de sistemas el comportamiento e interacción de las variables de la formación integral de los estudiantes, en cuanto a la ingeniería y el desarrollo de competencias.

Es importante recalcar que estos trabajos tienen en común el analisis del comportamiento sistémico de las variables en la educación, en tres enfoques posibles:

- Variables de producción de conocimiento de las instituciones y la relación con el entorno.

- Inclusión de la dinámica de sistemas o el pensamiento sistémico en determinados procesos de aprendizaje y formación en ingeniería.

- Análisis de las variables que se interrelacionan en los procesos de aprendizaje y formación de un individuo (análisis de competencias).

La incertidumbre del conocimiento se da en alta medida, ya que con la infinidad de fuentes, opiniones, teorías y fenómenos que transcurren en un corto tiempo, el conocimiento que hoy se construye y se transforma constantemente, es decir, el conocimiento es dinámico y complejo (Herrera \& Cortes, 2011). 
En el desarrollo de la investigación en Colombia confluyen tres actores que interactúan en los procesos de investigación: La Universidad, la Empresa y el Estado. Por una parte, la Universidad como la llamada a la construcción del conocimiento desde las Ciencias, la Empresa como el motor empírico donde se requieren las diferentes aplicaciones tecnológicas y desarrollos científicos, debido a la complejidad del entorno y a su llamado a la solución de problemas puntuales. Finalmente, el Estado como mediador de recursos, facilitador de políticas con el propósito de generar un ambiente propicio de investigación en una región.

La investigación como se ha planteado, debe tener un impacto social y humano que trascienda las fronteras de la Empresa y la Universidad. La investigación no es la excepción, no escapa a los retos de los contextos globales, y de igual manera debe transformar los discursos para soportar la permanente incertidumbre de que prodiga la información, la rápida movilidad del conocimiento, la creación de nuevas formas de lenguajes, respuestas a preguntas o problemas en tiempo record, esto de igual forma lleva a pensar en las alianzas interdisciplinares.

La Empresa en alianza estratégica con la Universidad puede lograr la innovación en propuestas funcionales de grado, pertinentes y contextualizadas, que sometan el conocimiento al servicio de la sociedad, permitiendo la productividad de las ideas en el plano significativo de la industria.

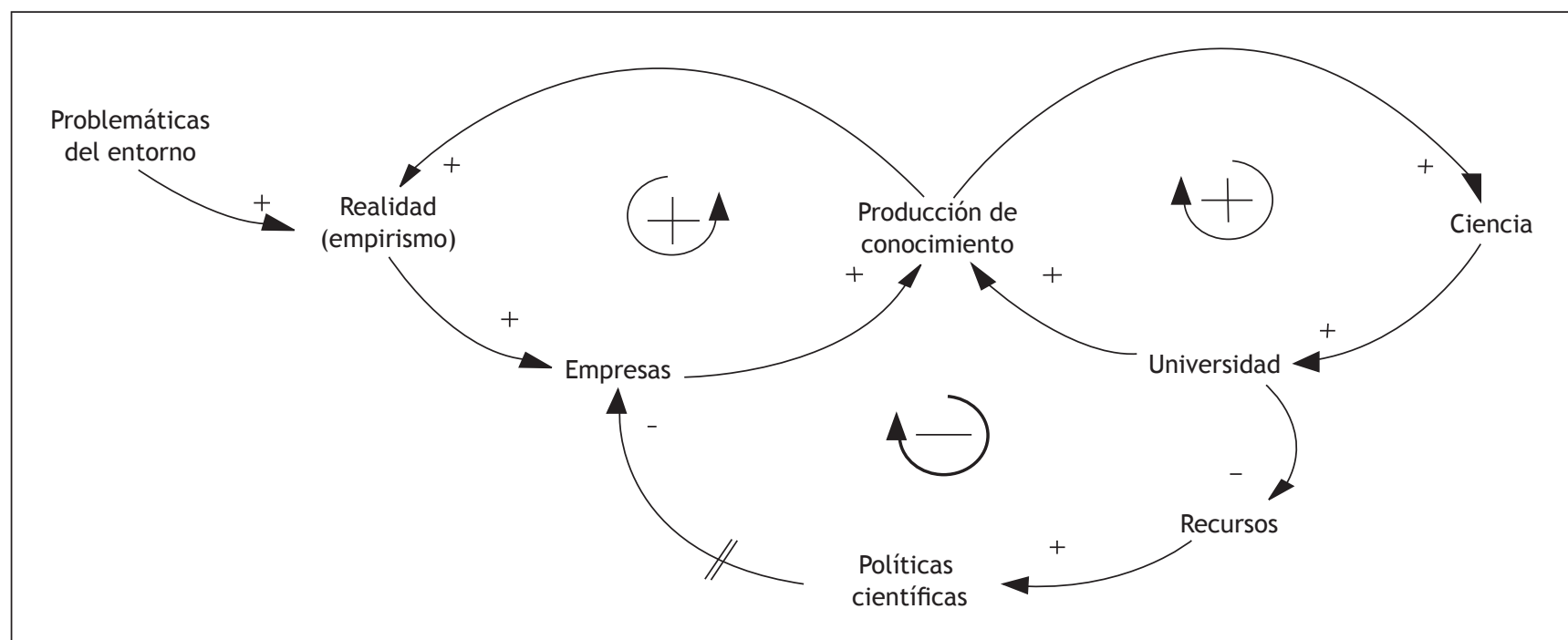

Figura 1. Modelo Causal de relación Universidad-Empresa-Estado. Fuente: Elaboración propia.

En la Figura 1 se puede observar como desde las problemáticas que nacen en el entorno y alimentan la realidad y las aplicaciones de las compañías, se produce el conocimiento enfocado a la resolución de problemas (Véase Herrera \& Cortes, 2011). Esta producción de conocimiento redunda en un aumento del conocimiento científico de las Universidades, que desde la propia ciencia también contribuyen al aumento de la producción de conocimiento. Sin embargo las limitaciones en los recursos (financieros, humanos etc.,) producen un efecto negativo en el proceso de difusión del conocimiento en las empresas, que nace en las universidades.

\section{CAPACIDADES DE CONOCIMIENTO EN LA UNIVERSIDAD - KNOW HOW}

Las universidades tienen la capacidad de generar conocimiento a partir del recurso humano que poseen, bien sea estudiantes a nivel de posgrados o pregrados (en menor medida) o docentes formados en el campo de la investigación. Este cúmulo de conocimiento producido en las universidades conlleva a determinar tiempos de dedicación para la generación de productos con impacto en la sociedad. Díaz et al. (2011) proponen un modelo causal de generación de conocimiento de la Universidad de los Andes basado en la formación de $\mathrm{PhD}$ y dedicación a la investigación de los estudiantes de doctorado (Véase Figura 2). Sin embargo la estrategia propuesta en el presente trabajo y desarrollada en la Universidad Piloto de Colombia, define como variables de generación del conocimiento la apropiación de los conocimientos y desarrollos existentes como semilla de programas de investigación estructurados y consolidados. 


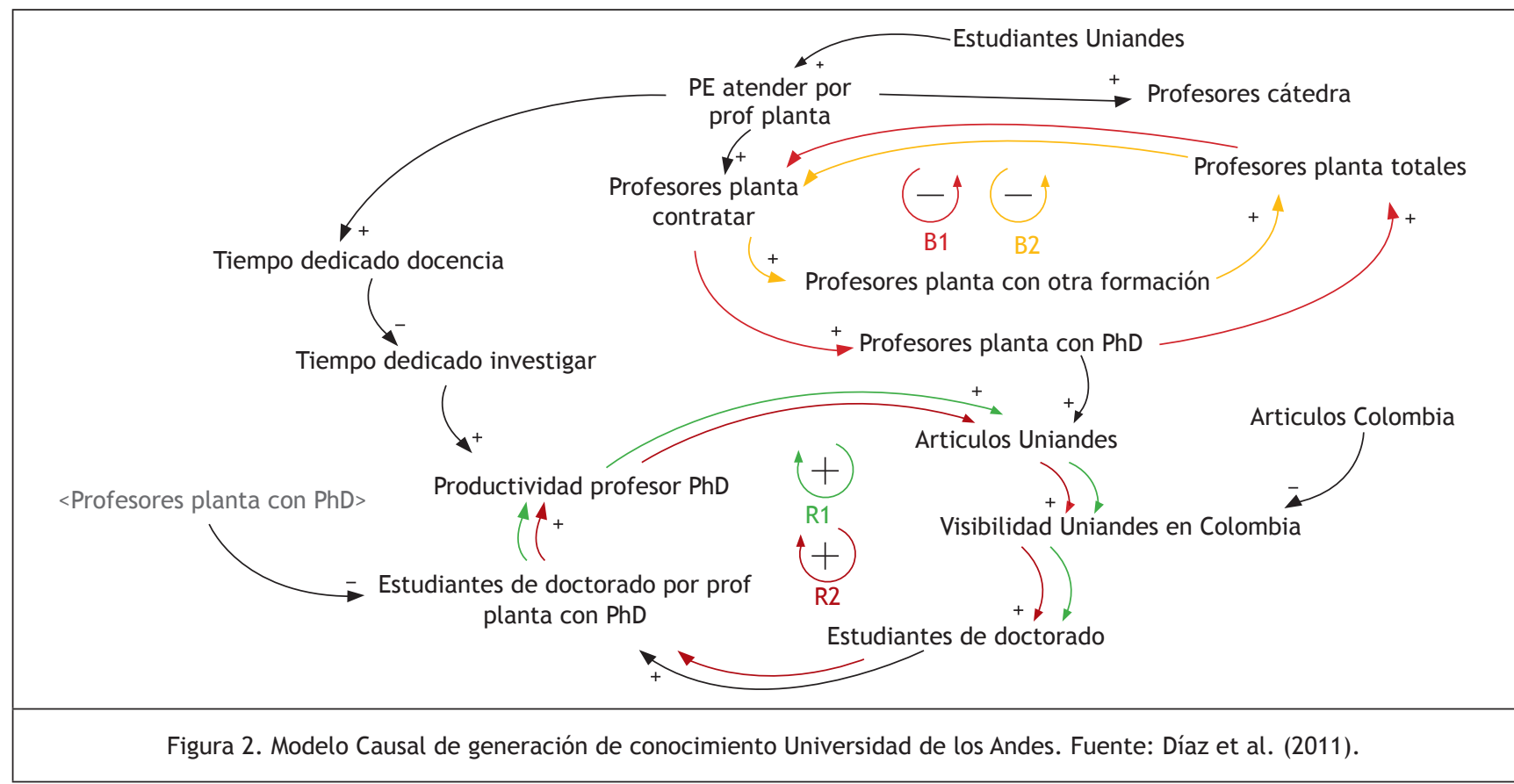

La estrategia de generación de conocimiento se plantea en el desarrollo y consolidación de áreas estratégicas requeridas en el mundo empírico (empresarial) o en las necesidades gubernamentales declaradas (planes de gobierno, estudios poblacionales etc.). Para el caso de aplicación se propusieron áreas estratégicas enmarcadas en temáticas orientadas en las líneas de investigación de la Universidad objeto de estudio y soportadas en las necesidades gubernamentales del Plan de Desarrollo para Colombia. Algunas de estas áreas se presentan a continuación:

- Desarrollo de modelos urbanísticos.

- Desarrollo de materiales sostenibles.

- Evaluación de servicios ambientales.

- Diseño de políticas públicas y regionales.

- Metodologías para el desarrollo de grupos de I+D+i de compañías.

- Modelos organizacionales y empresariales.

- Emprendimiento.

Seguidamente se plantea un análisis sobre la frecuencia en el desarrollo de este conocimiento con el fin de categorizar y jerarquizar el conocimiento (Know-How) adquirido en cada grupo de investigación. En la Figura 3 se puede observar la categorización y agrupación de la temática de ciudad dentro de los grupos de investigación de la Universidad Piloto de Colombia.

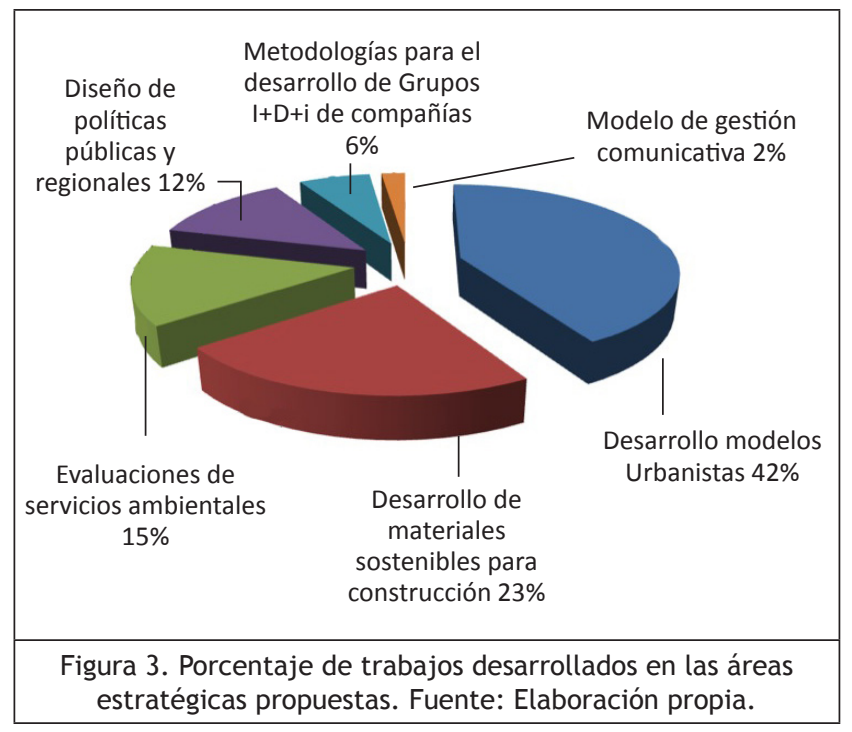

La frecuencia de los trabajos que se realizan en el desarrollo de modelos urbanísticos y materiales sostenibles presentan la mayor proporción y aunque con una menor proporción, la metodología para el desarrollo de grupos $I+D+i$, un tema transversal tratado en la gran mayoría de grupos de investigación.

\section{RELACIÓN DE CAPACIDAD DE APRENDIZAJE (KNOW-WHY) Y CONOCIMIENTO (KNOW-HOW)}

El desarrollo de las capacidades de aprendizaje (Know-Why) tanto de las universidades como de las 
compañías es adquirido en la medida en la que se obtiene conocimiento (Know-How) en el tiempo. La primera de estas (Know-Why) se relaciona con la mejora continua de los procesos de investigación desarrollados en las instituciones, mientras que la segunda (Know-Why) se fundamenta en el carácter innovador e impacto social que se refleja en la visibilidad y solución de problemas de la sociedad.

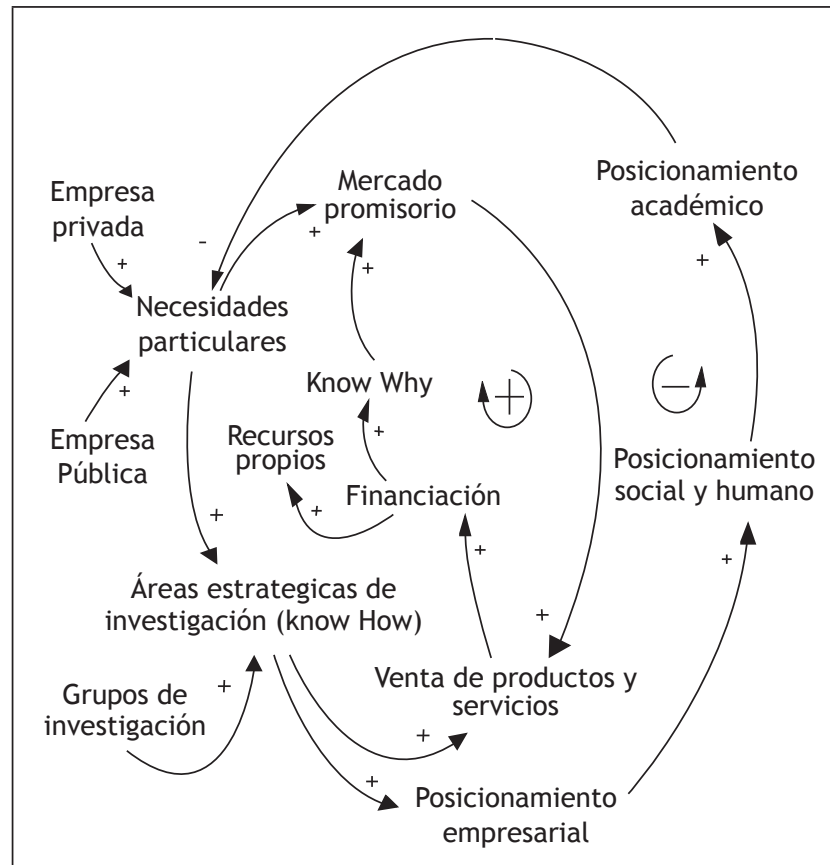

Figura 4. Modelo Causal de Estrategia de investigación propuesto. Fuente: Elaboración propia.

En la Figura 4 se observa un modelo causal de la estrategia de investigación propuesta, fundamentada en el desarrollo de áreas estratégicas basadas en el conocimiento de los grupos de investigación, desarrolladas a partir de las necesidades particulares de las compañías públicas y privadas. Las áreas estratégicas fundamentalmente proveen un efecto que genera un aumento en el portafolio de servicios y productos a ofrecer, y por ende un aumento en la financiación de la investigación y en el incremento de recursos propios. Estas relaciones causan un aumento en la capacidad de aprendizaje y por consiguiente mayor apropiación del entorno que conlleva la detección de mercados promisorios que surgen de las necesidades particulares.

Por otra parte, el bucle negativo de realimentación se fundamenta en la relación de competencia que se va generando desde las áreas estratégicas de investigación y el posicionamiento que se presenta a nivel empresarial, social, humano y académico, que disminuye las necesidades particulares de los actores (empresas públicas y privadas).
La relación entre las áreas estratégicas seleccionadas para determinados grupos de investigación debe estar orientada desde la dinámica del entorno, ya que este define el mercado promisorio a abordar. Si bien la investigación no se puede enfocar desde una perspectiva capitalista, sino por el contrario humanista que impacte la sociedad y el medio que nos rodea, esta se debe fundamentar en la consecución de recursos de inversión y desarrollo a través de políticas de investigación favorables.

\section{CONCLUSIONES}

Las relaciones causales propuestas desde la metodología de dinámica de sistemas proveen una visión holística de las posibles estrategias de investigación en las universidades. Algunas de estas políticas se proponen a continuación:

- Determinar áreas estratégicas de acuerdo con el Know How de la Universidad.

- Relacionar las áreas estratégicas encontradas con los posibles mercados promisorios de acuerdo con el entorno, revisando la capacidad de aprendizaje (Know Why) de la Universidad.

- Consolidación de recursos de investigación (grupos de investigación, conocimiento, finanzas etc.).

- Desarrollar políticas de incentivos para la búsqueda de recursos de financiación externa.

- Diseño de estrategias de construcción de programas de investigación con integración de los diferentes actores de investigación (UniversidadEmpresa-Estado).

- Desarrollo de estrategias de consecución de recursos y convenios de integración en proyectos orientados a la solución de problemas reales.

La consolidación de recursos de investigación propende por el mejoramiento institucional y el mejor aprovechamiento de los recursos, sin embargo las políticas externas propuestas desde el Estado deben incentivar el desarrollo de mecanismos favorables para la vinculación entre Empresa y Universidad. En esta vía durante los últimos años las entidades gubernamentales de los países en desarrollo han fomentado esta integración y fortalecimiento de las instituciones encargadas de regular las políticas de investigación en una región.

Finalmente, es de destacar que el incremento en las actividades investigativas y el favorecer las 
políticas encaminadas al desarrollo de los procesos investigativos redunda en el mejoramiento de la calidad de vida en una región, es por ello que el desarrollo de políticas de integración entre los actores de investigación confluye en satisfacer y dar solución a los problemas reales del ser humano.

\section{REFERENCIAS BIBLIOGRÁFICAS}

1. Abdelgawad A., Snaprud M. and Krogstie J. (2010). Accessibility of Norwegian Municipalities Websites: A qualitative System Dynamics Approach. Proceeding Conference The 28th International Conference of the System Dynamics Society.

2. Andrade, H., Navas X. and Maestre, G. (2010). Prácticas y aprendizajes con la dinámica de sistemas en la escuela Colombiana. Proceeding Conferences $8^{\circ}$ Congreso latinoamericano de Dinámica de Sistemas.

3. Andrade, H., Maestre, G. and López, G. (2011). Desarrollo de competencias en la toma de decisiones con dinámica de sistemas una experiencia en el aula. Proceeding Conferences $9^{\circ}$ Congreso latinoamericano de Dinámica de Sistemas.

4. Andersen, J., LaVigne, A., Radzicki, M., Richarson, G. and Stuntz, L. (2011). Teaching Charateristics of Complex Systems in K-12 Education. Proceeding Conference The 29th International Conference of the System Dynamics Society.

5. Arismendi, J. and Andrade, H. (2011). Reflexiones sobre el diseño de experimentos con dinámica de sistemas en educación. Proceeding Conferences $9^{\circ}$ Congreso latinoamericano de Dinámica de Sistemas.

6. Brammer, R. and Viehweger, B. (2010). The player nimber growth in Social Browser Games. Proceeding Conference The 28th International Conference of the System Dynamics Society.

7. Beall, A. and Ford, A. (2011). Learning from system dynamics simulations: time compression in system dynamic versus time dilation in power system simulators. Proceeding Conference The 29th International Conference of the System Dynamics Society.

8. Ballesteros, R. (2011). Competencias de los estudiantes de ingeniería de sistemas, análisis desde la dinámica de sistemas. Proceeding Conferences $9^{\circ}$ Congreso latinoamericano de Dinámica de Sistemas.

9. Castro, D., Jaén, S., Quiroz, A., Duque, S. and Ospina, D. (2010). En busca de la utopía: plasmando un ideal que puede ser real. Proceeding Conferences $8^{\circ}$ Congreso latinoamericano de Dinámica de Sistemas.

10. Cuménal D. (2010). How the concep of organisational value highlights corporate performance drivers a system dynamic teaching model. Proceeding Conference The 28th International Conference of the System Dynamics Society.

11. Davidsen, P., Bjurklo, M. and Wikstrom, H. (1993). Introducing system dynamics in schools: the Nordic experience. System Dynamic Review.

12. Díaz, A., Bucheli, V. and Zarama, R. (2010). Producción científica y acumulación de capital humano: un modelo para estudiar su relación en una universidad Colombiana. Proceeding Conferences $8^{\circ}$ Congreso latinoamericano de Dinámica de Sistemas.

13. Díaz, A., Bucheli, V. and Zarama, R. (2010). La investigación en la Universidad de los Andes como procesos de acumulación de capital humano: una aproximación desde la dinámica de sistemas. Proceeding Conferences $8^{\circ}$ Congreso latinoamericano de Dinámica de Sistemas.

14. Herrera, R. M., and Cortes, A. (2011). Metodología de la Investigación para resolver problemas en el entorno. Fondo Editorial Corporación Universitaria Minuto de Dios, Bogotá.

15. López, G. and Andrade, H. (2010). Los especialistas y la integración de la DS en la escuela una propuesta de aprendizaje colaborativo. Proceeding Conferences $8^{\circ}$ Congreso Latinoamericano de Dinámica de Sistemas. 
16. Maldonado, M. U., Maldonado, M., Cahim, R., Pietrobon, R. and Varvakis, G. (2010). University Industry partherships with a focus on BRIC countries: A system perspective on the good, the bad and the ugly. Proceeding Conference The 28th International Conference of the System Dynamics Society.

17. Richmond, B. (1993). Systems thinking: critical thinking skills for the 1990s and beyond. System Dynamics Review.
18. Ramírez, A., Robayo, J. and Sotaquira, R. (2011). Nuevas formas de inmersión para juegos de simulación en dinámica de sistemas. Proceeding Conferences $9^{\circ}$ Congreso latinoamericano de Dinámica de Sistemas.

19. Saeed, K. (1993). Bringing experimental learning to social science: simulation laboratory on issues of economic development. System Dynamic Review. 